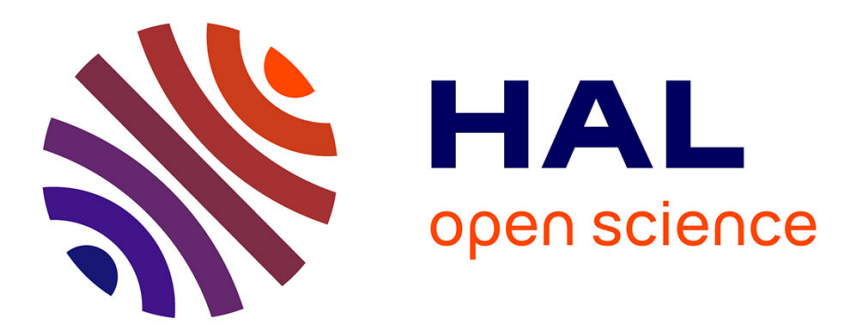

\title{
Diabetes remission after bariatric surgery in obese patients with haemochromatosis
}

F. Phan, C. Vatier, C. Vauloup-Soupault, Christine Poitou, J. Bouillot, J.

Oppert, J. Aron-Wisnewsky

\section{- To cite this version:}

F. Phan, C. Vatier, C. Vauloup-Soupault, Christine Poitou, J. Bouillot, et al.. Diabetes remission after bariatric surgery in obese patients with haemochromatosis. Diabetes \& Metabolism, 2017, 44 (2), pp.185-187. 10.1016/j.diabet.2017.02.005 . hal-01504520

\section{HAL Id: hal-01504520 \\ https: / hal.sorbonne-universite.fr/hal-01504520}

Submitted on 10 Apr 2017

HAL is a multi-disciplinary open access archive for the deposit and dissemination of scientific research documents, whether they are published or not. The documents may come from teaching and research institutions in France or abroad, or from public or private research centers.
L'archive ouverte pluridisciplinaire HAL, est destinée au dépôt et à la diffusion de documents scientifiques de niveau recherche, publiés ou non, émanant des établissements d'enseignement et de recherche français ou étrangers, des laboratoires publics ou privés. 
F. PHAN ${ }^{1,2}$, C. VATIER ${ }^{1,3,4}$, C. VAULOUP-SOUPAULT ${ }^{1,2}$, C. POITOU ${ }^{1,2,5} ;$ J.L BOUILLOT ${ }^{6}$; JM. OPPERT $^{1,2,7}$ J. ARON-WISNEWSKY $Y^{1,2,5}$

${ }^{1}$ Institute of Cardiometabolism and Nutrition, ICAN, Assistance Publique Hôpitaux de Paris,

${ }^{2}$ Nutrition department, Pitié-Salpêtrière Hospital, Nutrition department, F-75013, Paris, France;

${ }^{3}$ INSERM, UMR_S938, Centre de Recherche Saint-Antoine, Paris, France Sorbonne Universités, 75005

Paris, France

${ }^{4}$ Endocrinology Department Saint-Antoine hospital, Assistance Publique-Hôpitaux de

Paris, 75013 Paris, France

${ }^{5}$ Sorbonne Universités, UPMC Université Paris 06 and INSERM, UMR_S 1166, Team 6 Nutriomics, F75013, Paris, France;

${ }^{6}$ Visceral Surgery Department, Ambroise Paré Hospital, Assistance

Publique-Hôpitaux de Paris, 92100 Boulogne-Billancourt, France

${ }^{7}$ Paris 13 University, Sorbonne Paris Cité-Equipe de Recherche en Epidémiologie Nutritionnelle, U1153 Inserm, Inra, Cnam, Centre de Recherche en Epidémiologie et Biostatistiques; CRNH IdF, Bobigny, France.

Abbreviated tittle: Diabetes and hemochromatosis after bariatric surgery

Key terms: diabetes remission, cessation of phlebotomies, hemochromatosis, roux-en-Y gastric bypass, sleeve gastrectomy

Word count: 1729

\section{Number of figures and tables: 1}

\section{Corresponding author and person to whom reprint requests should be addressed:}

Judith Aron-Wisnewsky

Nutrition Department Pitié-Salpêtrière Hospital, Assistance Publique-Hôpitaux de Paris,

47-83 Boulevard de l'Hôpital

75013 Paris, France

email: judith.aron-wisnewsky@psl.aphp.fr

Disclosure Statement: The authors have nothing to disclose. 


\section{$\underline{\text { Introduction }}$}

Hemochromatosis represents a common autosomal recessive hereditary disease, inducing iron overload $^{1}$. The most frequent mutation results in a change from a cysteine to tyrosine at position 282 (C282Y) in the HFE protein. HFE is required for stimulation of Hepcidin, which acts as a regulator of iron intestinal absorption. When mutated, Hepcidin decreases and iron absorption remains inappropriately high, even when iron body stores are repleted ${ }^{2}$ Heme-iron is found in red meat, specifically in hemoprotein. After ingestion, iron is released from the heme secondary to low $\mathrm{pH}$ stomach and its enzymes. Most of iron absorption occurs in the duodenum and proximal jejunum. Iron enters the enterocytes through DMT-1 (ferrous iron transporter Divalent Metal ion Transporter-1), situated on the enterocyte brush border, and is exported into the blood, via a transmembrane protein called Ferroportin, found on the enterocytes basolateral membrane.

Hemochromatosis clinical symptoms appear progressively during adulthood such as secondary diabetes which treatments are identical to type-2 diabetes (T2D). The mechanisms of diabetes development during hemochromatosis involve both impaired insulin secretion and insulin-resistance; the latter can be exacerbated during obesity. Later complications include cirrhosis and hepatocellular carcinoma ${ }^{1}$. If therapeutic phlebotomy is the gold-standard treatment to reduce systemic iron levels, it does not play any beneficial role on glucose homeostasis ${ }^{1}$.

Obese patients often display hyperferritinemia, mostly related to liver metabolic alteration or obesityrelated low-grade inflammation. Sometimes however, the exploration of this hyperferritinemia may lead to hemochromatosis diagnosis. The number of bariatric surgeries (BS) performed has dramatically increased worldwide, since it allows major and sustained weight loss and marked metabolic comorbidities improvement $^{2}$. Importantly, 40-60\% of obese T2D patients achieve diabetes remission (DR), defined as glycemia and HbA1c normalization in the absence of T2D treatment, one year post-BS ${ }^{3}$. However, BS also induces nutritional deficiency ${ }^{4}$ due food intake decrease associated with a reduction of mineral and vitamin absorption especially after Roux-en-Y gastric bypass (RYGB). Therefore, obese patients without hemochromatosis are at risk of nutritional deficiencies (such as iron), that can be prevented by systematic multivitamin and iron supplementation ${ }^{4}$. Therefore, BS could represent an interesting therapeutic option for obese patients with hemochromatosis and diabetes. However, to date, if some case-reports document favourable changes in iron overload post-BS, there is currently no data on hemochromatosis induced diabetes evolution post-BS $\mathrm{S}^{5-8}$. Our aim is to report the evolution of both phlebotomies and diabetes treatment in 3 hemochromatosis patients with baseline diabetes who underwent BS.

\section{Patients \& Methods}

Patient A was already diagnosed with hemochromatosis before BS. In patients B and C, hemochromatosis screening was performed because of biological iron overload evidenced during pre-operative assessment. Clinical and biological data (weight, phlebotomy frequency, transferrrin saturation, ferritin, anti-diabetic 
treatments evolution and $\mathrm{HbAlc}$ ) were retrospectively gathered before surgery and at each follow-up visit until the last available post-BS follow-up (Table 1). Ethical approval was obtained from the Research Ethics Committee of Hotel-Dieu Hospital (CPP Ile-de-France_1).

\section{$\underline{\text { Results }}$}

The 3 patients, with hereditary hemochromatosis also present morbid obesity associated with either $\mathrm{T} 2 \mathrm{D}$ or pre-diabetes. Besides hemochromatosis, all 3 patients displayed the required BS indications.

Patient A was diagnosed with heterozygous hemochromatosis (C282Y/H63D) in the context of porphyria cutanea at 32 years old, in 2001. He developed secondary diabetes, 9 years later which was treated with metformin, glimepiride and subsequently in 2010 with bed-time insulin due to increased HbA1c. Phlebotomy was initiated at diagnosis and continued once a month for 9 years, until he underwent RYGB in $2010\left(\mathrm{BMI}=42 \mathrm{~kg} / \mathrm{m}^{2}\right)$. BS enabled a substantial weight reduction, maintained until the last follow-up, 6 years post-BS. Phlebotomy sessions were stopped immediately post-RYGB and such is still true in 2016. Since there was too few data in the literature to guide the treatment, the patient initially received iron supplements for the first 5 months, which was stopped thereafter for the remaining follow-up. The patient underwent complete DR at one year and is still in prolonged remission in $2016^{3}$.

Patient B was diagnosed with homozygous hemochromatosis (C282Y) at 43 years old in 2010, and presented pre-diabetes. At diagnosis, phlebotomies were started every 3 weeks for 2 years. RYGB, performed in $2012\left(\mathrm{BMI}=43.8 \mathrm{~kg} / \mathrm{m}^{2}\right)$, enabled substantial weight loss, which was maintained during 4 years of followup. She was never prescribed iron supplementation post-BS. Phlebotomy sessions were continued for 6 months post-RYGB and then stopped for the remaining follow-up with normalization of iron load. Glucose homeostasis normalized as soon as 3 months post-RYGB and has remained normalized ever since until 2016.

Patient $\mathrm{C}, 45$ years old, was diagnosed with homozygous hemochromatosis (C282Y) three years before undergoing sleeve gastrectomy (SG). Although RYGB was more indicated in such medical situation, SG was performed according to patient's will and surgery feasibility. His baseline diabetic treatment consisted in metformin, glimepiride and sitagliptin. Phebotomy was performed every 5 weeks for 3 years. $\mathrm{SG}$, performed at BMI $=57.1 \mathrm{~kg} / \mathrm{m}^{2}$, enabled marked weight reduction during the first year with a slight weight regain at the end of the follow-up. By contrast to the others, patient $\mathrm{C}$ still underwent phlebotomy during 2 years post-SG due to persistent hyperferritinemia despite no iron supplementation during followup. Phlebotomies were first staggered then completely stopped at 2 years post-SG. Glucose homeostasis progressively improved post-SG with a gradual reduction of anti-diabetic treatments. The patient entered partial DR one year post-SG (i.e $6 \mathrm{mmol} / 1<$ glycemia $<7 \mathrm{mmol} / \mathrm{L}$ without anti-T2D treatment) ${ }^{3}$. 


\section{Discussion}

We herein display long term result of hemochromatosis patients with T2D undergoing BS with beneficial effects on both diseases. Indeed, in parallel with weight loss and DR post-BS, phlebotomies, the only effective hemochromatosis treatment nowadays, were stopped. Our study adds to the current knowledge, since we display the longest post-BS follow-up study of hemochromatosis patients with diabetes. Indeed, literature only revealed 5 cases of hemochromatosis patients with obesity, who underwent RYGB, with a follow-up ranging from 10 to 60 months ${ }^{5-8} .3$ males and 2 females aged from 40 to 55 years old, with a BMI between 41.4 and $59.9 \mathrm{~kg} / \mathrm{m}^{2}$, were treated with phlebotomies every 2 weeks ${ }^{7}$ to every 3 months ${ }^{5,6}$ for hereditary hemochromatosis before their RYGB. Phlebotomies were stopped between 6 months $^{6}$ to 3 years ${ }^{5}$ post-RYGB except for one patient who still underwent phlebotomies twice a year at the end of the described follow-up of 4.5 years $^{8}$. If those previous case-report already displayed beneficial outcomes on phlebotomies cessation post-BS, they did not provide regular kinetic data regarding iron metabolism biology nor any information on potential mineral supplementations/cessation post-RYGB. Furthermore, none of these reported cases had secondary diabetes prior to BS. Herein, we were able to gather short and the longer-term kinetic data yet available in the literature, in terms of phelobotomy cessation but also on iron metabolism biomarkers and iron supplementation. By contrast to previously published data, we observe early phlebotomy cessation post-RYGB as soon as 1 month for patient A, although he had a long past history of diagnosed hemochromatosis and 6 months post-RYGB for patient B with more recent diagnosis. Interestingly, we also report later phlebotomy cessation post-sleeve gastrectomy.

The underlying mecanisms explaining why phlebotomies can be stopped includes several factors influencing iron absorption post-BS. Firstly, dietary intake, and particularly meat consumption, thus iron intake, decreases drastically due to smaller stomach ${ }^{4}$, thus reducing dietary iron availability. Secondly, gastric volume reduction decreases gastric secretions, subsequently leading to gastric $\mathrm{pH}$ increase. Since acidity allows iron release from heme, its solubilization and ionization, BS leads to reduced iron absorption ${ }^{4}$. Thirdly, since the most important site of iron absorption takes place in the duodenum and proximal jejunum which are bypassed in RYGB, it is further reduced after this specific intervention ${ }^{5}$. Indeed, using an isotopic method $^{9}$, iron absorption was assessed post-RYGB and it was demonstrated that patients could not increase their iron absorption despite overt iron-deficient anemia post-RYGB.

Importantly, we also report herein an additional effect on T2D improvement. Indeed, none of the previously published case-reports had T2D, by contrast to our series with either diabetes or prediabetes, which could be either T2D or secondary hemochromatosis diabetes. All patients displayed DR which can likely be due to concomitant weight loss and malabsorption-induced iron depletion. Patient A was diagnosed with diabetes 9 years after the diagnosis of porphyria cutanea despite already efficient phlebotomy iron depletion. Subsequently, T2D worsened as evidenced by the increase in anti-diabetic treatment before BS. However, anti-diabetic treatments could be completely stopped 6 months post-surgery and the patient is still in prolonged DR after 6 years, although the DiaRem $^{10}$, a valid score for baseline DR prediction, did not 
anticipate T2D remission. Indeed, 4 parameters (age, $\mathrm{HbA1c}$, antidiabetic medication and insulin treatment) with different weight are used to develop this score, which ranges from 0 to 22 . A high score is associated with non diabetes remission, which is the case of Patient A. This suggests that in this patient, the added effect of iron normalization played a major role in DR.

Likewise, patient B was diagnosed with pre-diabetes, 2 years before BS. Due to efficient phlebotomy, pre-diabetes improvement occured 3 months post-BS, with subsequent normalization of glycemic parameters for the following 4 years.

Conversely, patient $\mathrm{C}$, operated from SG displayed a less rapid and efficient resolution of both iron overload and glucose homeostasis. Several explanations are possible. SG does not have the additive iron malabsorption mechanism seen in RYGB. Furthermore, although SG also improves T2D, a randomized trial showed that HbA1c reached target goals, yet at the cost of more patients remaining under antidiabetic treatments after sleeve as compared to $\mathrm{RYBG}^{2}$. Of note, diabetes was more severe in this patient, as seen with high HBA1c, which might also explain the increased delay of DR.

Importantly, one major pitfall of T2D improvement post-BS, is its frequent recurrence in the longer term $^{2}$. Yet, none of our patients displayed diabetes recurrence thus again suggesting that iron normalization was involved in glycemic control achievement in these hemochromatosis patients.

\section{$\underline{\text { Conclusion }}$}

Overall, we report that BS in obese patients with hemochromatosis and diabetes enable weight loss and phlebotomy cessation that can be maintained in the long term, thus entering in prolonged remission. Iron status normalization is due to both decreased food intake and malabsorption, whereas diabetes resolution probably originates from both weigth loss and iron normalization. In addition, we highlight a differential effect of two BS technics with faster beneficial outcomes post-RYGB in terms of phlebotomy cessation and DR, maybe due to the added iron malabosption. We aim to increase the number of study patients and the follow-up duration to assess more comprehensively the overall benefits of BS in obese patients with hemochromatosis and diabetes.

\section{REFERENCES}

1. Powell LW, Seckington RC, Deugnier Y. Haemochromatosis. Lancet Lond Engl. 2016 Mar 11;

2. Schauer PR, Mingrone G, Ikramuddin S, Wolfe B. Clinical outcomes of metabolic surgery: efficacy of glycemic control, weight loss, and remission of diabetes. Diabetes Care 2016;39:902-911 
3. Buse JB, Caprio S, Cefalu WT, Ceriello A, Del Prato S, Inzucchi SE, et al. How do we define cure of diabetes? Diabetes Care. 2009 Nov;32(11):2133-5.

4. Verger EO, Aron-Wisnewsky J, Dao MC, Kayser BD, Oppert J-M, Bouillot J-L, et al. Micronutrient and Protein Deficiencies After Gastric Bypass and Sleeve Gastrectomy: a 1-year Follow-up. Obes Surg. 2016 Apr;26(4):785-96.

5. Kudva GC, Bacon BR. Iron deficiency anemia in hereditary hemochromatosis after gastric bypass surgery. Ann Hematol. 2009 Mar;88(3):291-2.

6. Gopaluni S, Martinez-Balzano C, Graziano S. Gastric bypass surgery as an intervention for obese patients with hemochromatosis. QJM Mon J Assoc Physicians. 2014 Dec;107(12):1023-5.

7. Ott M, Stouffer C, Foote J, Foster A. Roux-en-Y gastric bypass: a novel approach to the treatment of hemochromatosis? Am J Hematol. 2007 Nov;82(11):1033.

8. Barton JC. Hemochromatosis, HFE C282Y homozygosity, and bariatric surgery: report of three cases. Obes Surg. 2004 Dec;14(10):1409-14.

9. Ruz M, Carrasco F, Rojas P, Codoceo J, Inostroza J, Basfi-Fer K, et al. Heme- and nonheme-iron absorption and iron status 12 mo after sleeve gastrectomy and Roux-en-Y gastric bypass in morbidly obese women. Am J Clin Nutr. 2012 Oct;96(4):810-7.

10. Still CD, Wood GC, Benotti P, Petrick AT, Gabrielsen J, Strodel WE, et al. Preoperative prediction of type 2 diabetes remission after Roux-en-Y gastric bypass surgery: a retrospective cohort study. Lancet Diabetes Endocrinol. 2014 Jan;2(1):38-45. 


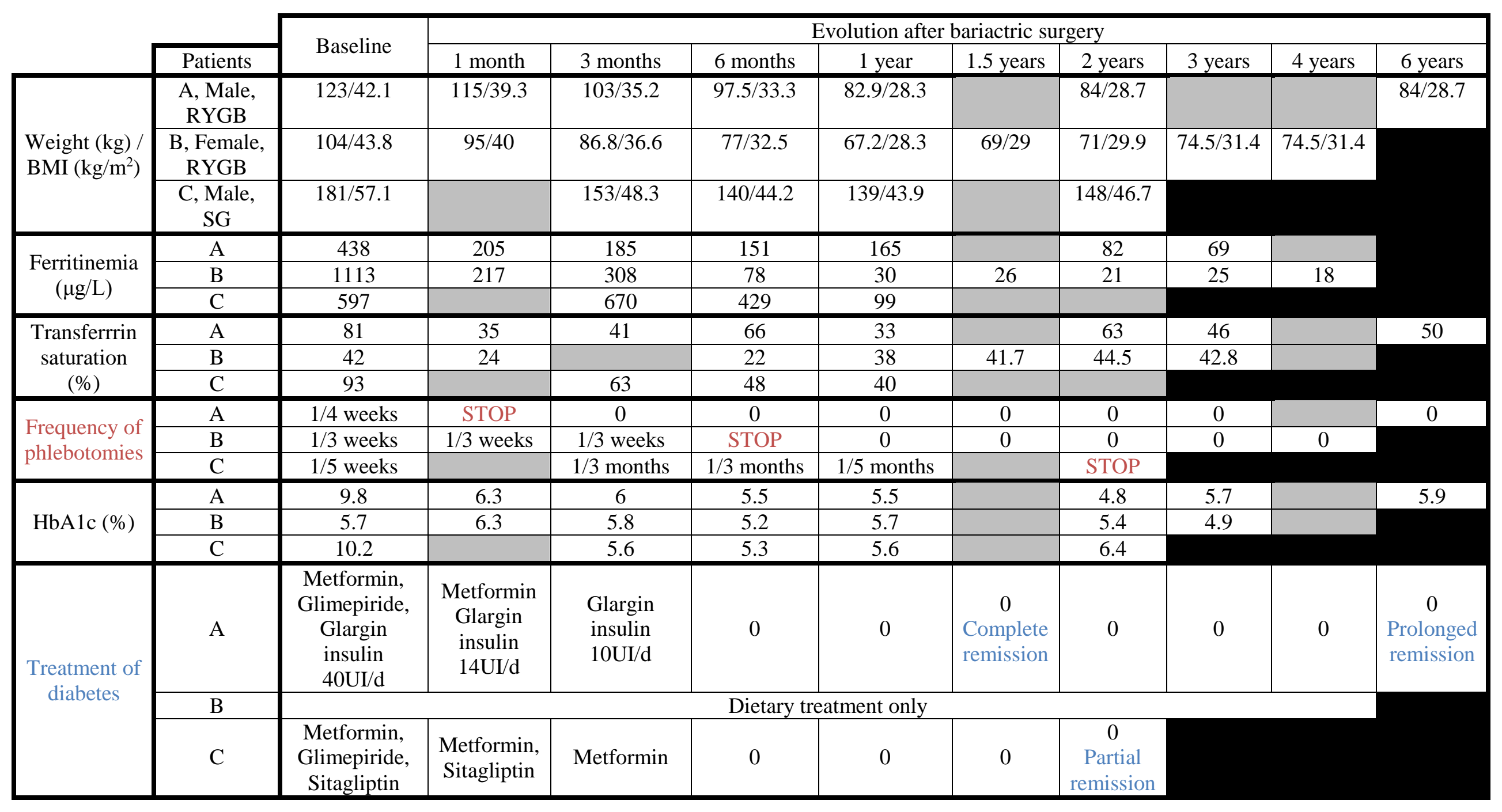

$$
\text { Missing data } \quad \text { No follow-up to this date }
$$

